\title{
Journal of Foot and Ankle Research
}

Oral presentation

Open Access

\section{Direct in vivo quantification of the 3D talocrural and subtalar finite helical axes Frances T Sheehan}

Address: Physical Disabilities Branch, National Institutes of Health, Bethesda, MD, USA

Email: Frances T Sheehan - fsheehan@cc.nih.gov

from Ist Congress of the International Foot \& Ankle Biomechanics (i-FAB) community

Bologna, Italy. 4-6 September 2008

Published: 26 September 2008

Journal of Foot and Ankle Research 2008, I (Suppl I):O35 doi:I0.I I86/I757-I I46-I-SI-O35

This abstract is available from: http://www.jfootankleres.com/content/I/SI/O35

(c) 2008 Sheehan; licensee BioMed Central Ltd.

\section{Introduction}

In an attempt to understand the etiology of ankle joint injury and degeneration, numerous models have been created as a means to estimate ankle joint forces. Due to a lack of non-invasive in vivo measurement techniques, the kinematics required to drive these models have typically been acquired in cadavers or using external markers to infer internal bone motion. This has left some uncertainty as to the validity of these models. Thus, the purpose of this study was to non-invasively quantify the $3 \mathrm{D}$ in vivo finite helical axes (FHA) of the subtalar and talocrural joints.

\section{Methods}

Twenty healthy subjects $(25.9 \pm 4.1$ years, $70.3 \pm 12.8 \mathrm{~kg}$, $174.1 \pm 7.7 \mathrm{~cm}$ ) participated in this IRB approved study. Subjects were placed supine in a $1.5 \mathrm{~T}$ MR imager (LX; GE Medical Systems, Milwaukee, WI, USA) after obtaining informed consent. An MRI-compatible device was used to apply a plantarflexion (PF) load. This device allowed natural 3D motion at both joints of the hindfoot. While subjects cyclically plantar-flexed and dorsiflexed their ankle at 35 cycles/min, aided by an auditory metronome, fast-PC $M R$ images (anatomic and $x, y$, and $z$ velocity images, temporal resolution $=72 \mathrm{~ms}$, imaging time $=2: 48$ ) were collected [1]. The sagittal-oblique imaging plane contained the tibia, calcaneus, and talus. The 3D time dependent tibial, talar and calcaneal attitudes were quantified through integration of the velocity data [1]. From these data the FHAs were determined.

\section{Results}

The ratio of the talocrural to subtalar angular velocities varied greatly between subjects. This ratio ranged from 1.1 to 4.5 . Talocrural joint rotation occurred primarily about the medial-lateral axis (Figure 1A and 1C) during plantarflexion. Yet the FHA was not oriented solely in the M-L direction and rotation of talocrural FHA with minimal displacement during PF was seen across all subjects. The subtalar FHA was directed primarily in the anterior-posterior direction (Figure 1B and 1D). The amount of angulation in the M-L and superior-inferior directions varied during PF and was variable across subjects.

\section{Conclusion}

The data from this study clearly confirmed that neither joint is a simple hinge joint and that ankle rotation arose primarily from the talocrural joint. The tendency of the subtalar and talocrural FHAs to rotate and translate will impact the calculation of tendon and ligament moment arms and, thus, alter the moment producing capabilities of the force generating structures at the ankle joint. Future modelling studies should investigate the sensitivity of the model outputs to variations in the FHA direction and location. 

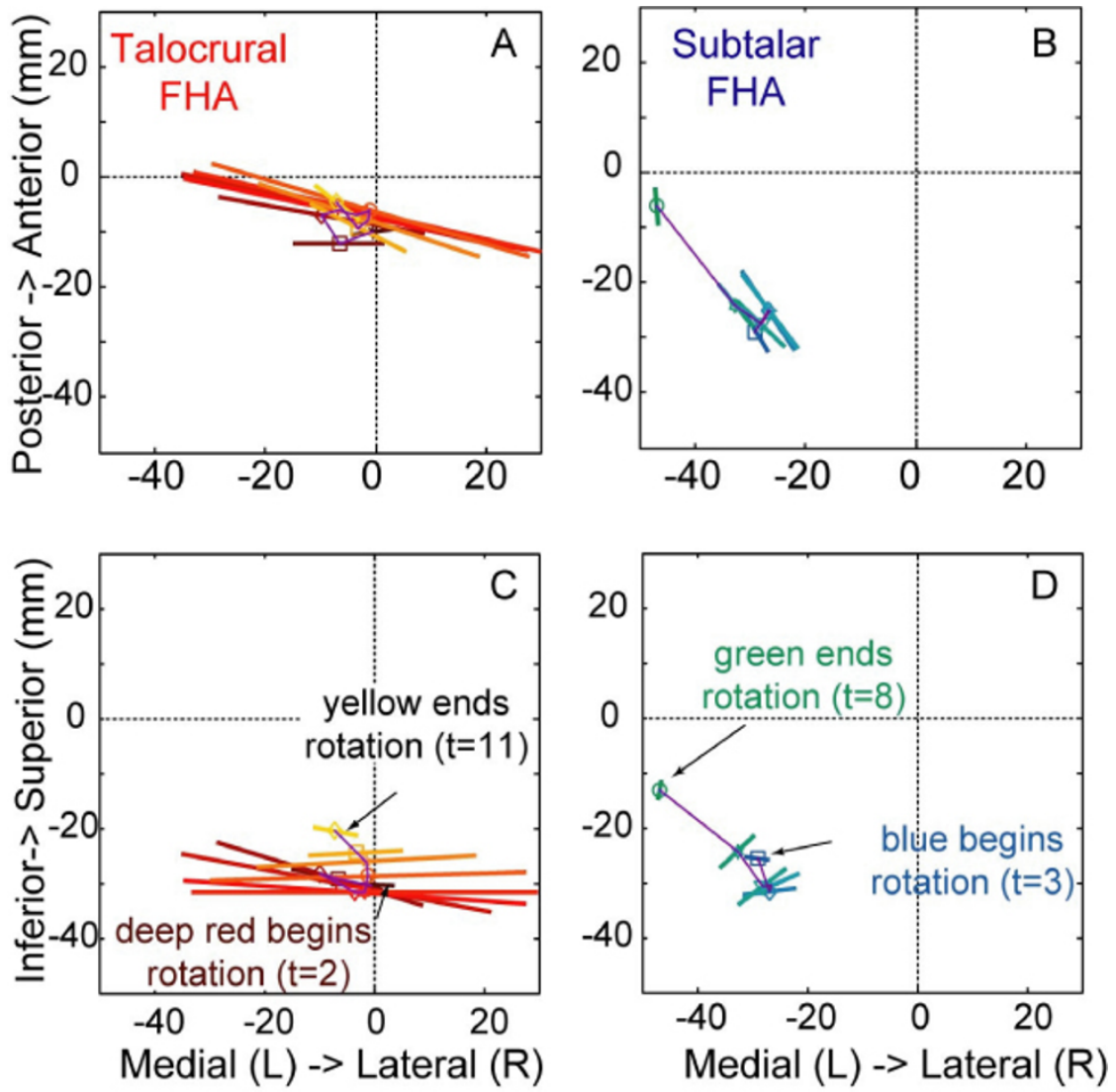

\section{Figure I}

FHAs for subject 982IR. All FHA's are plotted relative to the tibial coordinate system. The talocrural (subtalar) FHA changes from deep red (blue) to yellow (green) as the ankle plantarflexes. The length of the FHA indicates the magnitude of the angular velocity with the center of the line being the closest point the tibial origin (A\&C) and talar origin (B\&D). Ankle angle for this subject ranged from $1.9^{\circ}$ to $39.4^{\circ}$.

\section{References}

I. Sheehan FT, et al.: Foot \& Ankle Int 2007, 28(3):323-3. 